\title{
DIABETES
}

\section{Pre-eclampsia: association with increased risk of diabetes}

A population-based study of $>1$ million women has found that women with preeclampsia or gestational hypertension have a twofold increased risk of developing diabetes after pregnancy. Denice Feig and colleagues also found that the presence of pre-eclampsia or gestational hypertension together with gestational diabetes was associated with a further elevation in diabetes risk, beyond that seen with gestational diabetes alone. "These findings highlight a possible new risk factor for diabetes, and support the need to counsel patients with hypertensive disorders of pregnancy regarding postpartum diabetes screening prevention," say the researchers.

Gestational diabetes is a known risk factor for the future development of diabetes. "Since insulin resistance is a key component of diabetes, we wondered whether pre-eclampsia and gestational hypertension, which are also associated with insulin resistance, would increase an individual's risk of developing diabetes," explains Feig.
In their retrospective cohort study, Feig et al. used hospital records and outpatient data to categorize 1,010,068 women who gave birth in Ontario, Canada between 1994 and 2008, as having either preeclampsia, gestational hypertension, gestational diabetes, or a combination of these disorders. Women were followed until 2011 and assessed for the development of diabetes.

\section{These findings ... support the need to counsel patients with hypertensive disorders of pregnancy... 77}

Over a median follow-up of 8.5 years (range 1 day to 16.5 years), the researchers identified 22,933 cases of pre-eclampsia alone, 27,605 cases of gestational hypertension alone, 30,852 cases of gestational diabetes alone, 2,100 cases of gestational diabetes plus gestational hypertension, and 1,476 cases of gestational diabetes plus pre-eclampsia. 35,077 (3.5\%) of women developed diabetes.

After adjusting for various factors, both pre-eclampsia and gestational hypertension alone were identified as risk factors for the development of diabetes (hazard ratios [HRs] 2.08 and 1.95 , respectively). Gestational diabetes markedly increased the risk of developing diabetes (HR 12.77), and the presence of pre-eclampsia or gestational hypertension in addition to gestational diabetes increased this risk further (HRs 15.75 and 18.49, respectively). "By identifying future risks in women with disorders during pregnancy, we have a perfect opportunity to develop strategies to intervene early in these processes in order to prevent future disorders from developing," says Feig.

Susan J. Allison

Original article Feig, D. S. et al. Preeclampsia as a risk factor for diabetes: a population-based cohort study. PLOS Med. 10, e1001425 (2013) 